\title{
Bottomless Taxonomy of Cooperative Design for Flying Ad Hoc Network
}

\author{
Sandeep Raj, V.K. Panchal, Rajiv Chopra
}

\begin{abstract}
A framework of a network explains the application of the system. In cooperative framework design, model and architecture is the issued context of dynamic topology. The topology of the network continuously changes when the nodes are moving. There are different topologies and routing protocols that are running frontally and at backsides considered in the paper. We have done deep analysis on various protocols and network architecture of UAVs (unmanned aerial vehicle). Some different characteristics have been taken to explain the unmanned aerial vehicle as example. There are multiple factors and different scenarios that acknowledge the operability of cooperative network. In addition, the paper has been designed in such a way that it defines different aspects of cooperative networks. Collaboration and cooperation are the networking tasks for the multiple groups of nodes that combine and provide a reliable connectivity between the nodes. Flying ad hoc network (FANET) is the same name of unmanned aerial vehicle that are flying with the dynamic environment and can identify the ground node to send information. This is important task for the aerial nodes that are continuously flying in the air and send data collected from particular area. So, every task is important for accomplished task such as coordination and cooperation of all the nodes. This paper overall has focused on deep analysis of the taxonomy for unmanned aerial vehicle in the real environment.
\end{abstract}

Keywords: Environment, unmanned aerial vehicle, cooperation network, flying ad hoc network

\section{INTRODUCTION}

Cooperative interaction and association are the evolving fields in ad hoc networking nowadays. The execution of ad hoc has broadened the scope in the area of networks [1-3]. Temporary network comprised of nub which can perform routing and data calculations before sending it to other connected nub, this process is known as relaying. This network comprises of various transmitters, receivers and transferring nub. The cooperation is most important task in an unmanned aerial vehicle. Unmanned aerial vehicles (UAVs)

Revised Manuscript Received on April, 022020.

* Correspondence Author

Sandeep Raj*, department of Computer Science \& Engineering, Al-Falah University, Faridabad, Haryana, India. Email: sandeepbainy01@gmail.com

Dr. V.K Panchal, department of Computer Science \& Engineering, Al-Falah University, Faridabad, Haryana, India. Email: vkpans@gmail.com

Dr. Rajiv Chopra, department of Computer Science \& Engineering, Guru Tegh Bahadur Institute of Technology, affli. to GGSIPU, New Delhi, India. Email:raj_74chopra2004@yahoo.com

(C) The Authors. Published by Blue Eyes Intelligence Engineering and Sciences Publication (BEIESP). This is an open access article under the CC BY-NC-ND license (http://creativecommons.org/licenses/by-nc-nd/4.0/) are defined as aerial vehicles that can fly independently and form networks in the sky used for data transfer to the ground stations. Single UAV system is also developed that can be seen in the form of drones. These systems don't have ad hoc formation and have to be dependent upon satellite communication [5]. However, these UAVs have a restriction for surveillance and security, also in case of failure there is a complete disability in-network with no chance of recovery. These problems are resolved with ad hoc formation in UAVs, termed as flying ad hoc network (FANETs).

In ad hoc network every UAV can reply independently; also UAVs can form systems that are UAV swarms to extend the network monitoring [4-7]. In most recent studies by Bekmezci et al. [2], Unmanned aerial vehicle system has been freely contemplated as flying ad hoc system.

Aerial impromptu creation [8-9] is likewise executed as of now; however, the significant distinction is the self-governing vehicles and the necessity to share algorithms and information to evade the chances of any collisions among vehicles. This literature analysis of survey considered for aggregate important points related to UAVs includes 38 papers.

\section{MOTIVATION}

The important property in the UAVs is to perform correct coordination and cooperation between the various airborne nodes. The existing study or survey cannot provide an insight into these models for their networking features and their cooperative framework.

Thus, there exists a need for an independent study of these models to provide a detailed insight with their features and highlights, and to enable the pollsters to recognize the hypothetical related issues of networking with the aerial vehicles and also provide a careful investigation of existing methodologies to identify the solutions. In this paper, we have focused on the multi-UAV models and the formation of multi-UAV cooperative systems.

\section{BACKGROUND}

Unmanned aerial vehicles (UAVs) are independent components that are capable of utilization of controlled conditions to provide structure in air. These types of systems are named as aerial ad hoc networks depicted in Fig. 1(a), 1(b).

Such a system arrangement diminishes any necessity of satellite correspondence, consequently lessening the expense of system development [12]. UAVs are categorized in two ways: 


\section{Bottomless Taxonomy of Cooperative Design for Flying Ad Hoc Network}

(1) Infrastructure based ad hoc networks: It uses devices operated from the ground station. Such systems are a mixture of MANETs and VANETs [1]. These networks combining systems could deliver prolonged susceptibility and more error-free reducing paths.

(2) Cooperative based networks: It is multi-UAVs based systems forming homogenous and heterogeneous structures as shown in Fig. 1(b). Such system involves a UAV forming an ad hoc network, operated from ground station acting as input / output unit for shared information and an interface controlling the data flow among the multiple nodes.

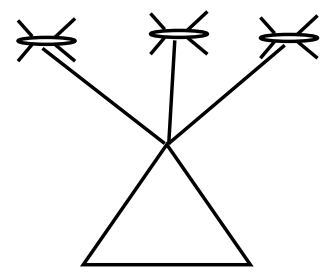

Fig. 1. (a) Arrangement of ad hoc network

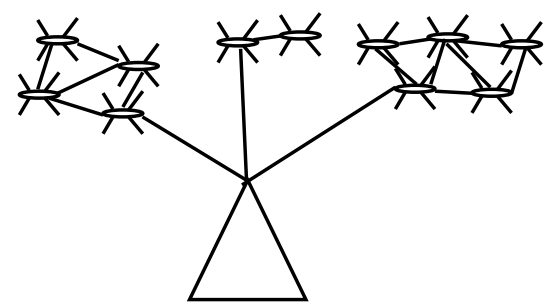

Fig. 1. (b) Arrangement of cooperative network

This flow of information in this network is known as routing cooperative framework. Such network framework enables the complex tasks in networking [11]. Routing is used to provide better information tracking and data attainment among the nodes [10].

\section{CHARACTERISTICS}

Unmanned aerial vehicles have a wide range of characteristics present in current scenario. There are some main characteristics that are depicted in Fig. 2, and can be described as following:

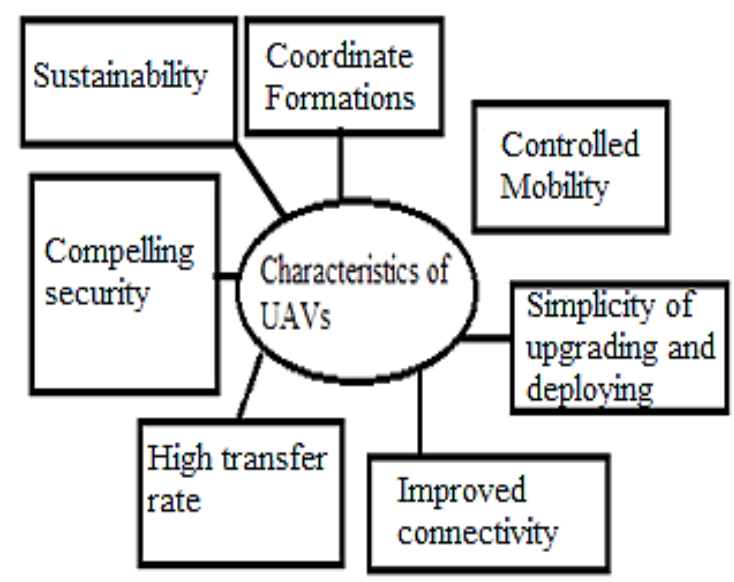

Fig. 2. Representation of characteristics

\subsection{Coordinate formations}

UAVs have the ability to perform independent operations and collaborative network formations. The availabilities of different known bands in the networking for wireless communication; UAVs can use these bands for the coordinate from the distance with the ground stations as well as handle satellite based communication simultaneously.

\subsection{Controlled mobility}

The transmission of data can be controlled effectively and can function well during the controlled environment. With such controlled networks, these air vehicles find their place in wide areas of civilian and military activities.

\subsection{Simplicity of upgrading and deploying}

Independent of the platform formation, these networks are easy to be upgraded and maintained. Nodes can be easily configured at any time during the operation and are also easy to be deployed.

\subsection{Improved Connectivity}

As compared to Mobile Ad Hoc Networks (MANETs) and Vehicular Ad Hoc Networks (VANETs), these are less few vulnerable to network loss as bandwidth pertinent to such network provides fewer obstacles.

\subsection{High transfer rate}

There is a high bandwidth which provides lesser delays and improved connection between the nubs. After the network is established it will provide a higher transfer rate.

\subsection{Compelling security}

Higher bandwidth provides the easy spread of the spectrum without reallocation of channels which makes it easy to maintain the corridor privacy in such networks and provide efficient security application.

\subsection{Sustainability}

These networks are able to survive the unfavorable condition because of certain improvement in deployment algorithms. In a solitary network multiple UAVs can be utilized for various functions without the rearrangement of the entire system. The above mentioned attributes shows, these systems give scope of utility and various functions in diverse situations.

\section{APPLICATIONS}

Ad hoc network delivers an extensive range of functions and applications. Some of these applications are mentioned below:

a) Military expeditions

b) Mutual formation of network

c) Natural calamities

d) Sudden crisis encounter

e) Continuous patient monitoring

f) Detection of security suspension

The top most companies that are manufacturing Unmanned Aerial Vehicles depicted in Fig. 3. These companies have manufactured the aerial vehicle for the specific tenacities used for a capacity improvement of prevailing networks for delivery services; for example, Amazon [13] has used delivery and scheduling services to provide supply at doorsteps with the help of drones.

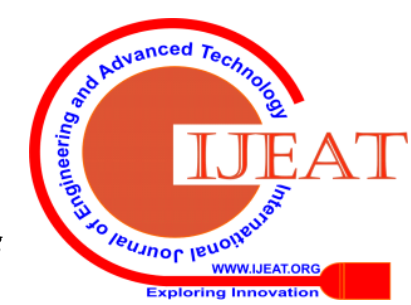




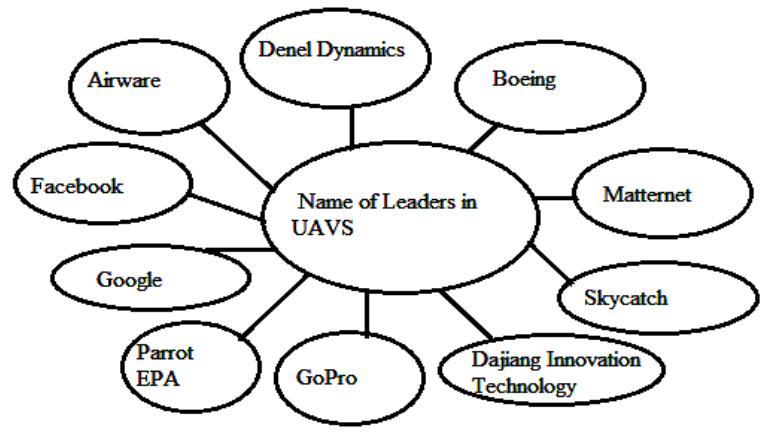

Fig. 3. Manufacturing Companies of UAVs

\section{CHALLENGES OF AERIAL VEHICLE ARRANGEMENT}

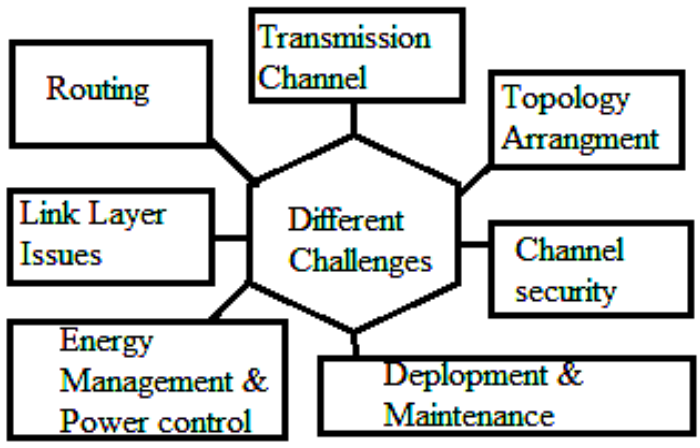

Fig. 4. Challenges in Aerial Vehicle

\section{(a) Topology}

All connected nodes are organized in such a way that increases the performance of the networks in an efficient manner. Revival and rearrangement at certain points are some important aspects of topology in cases of network failures [14-15].

\section{(b) Transmission Channel}

As aerial networks mostly provide a line of sight so, it is difficult to choose a suitable band for transmission in networks having multiple nodes. Likewise, to provide higher transfer rates this selection has to be carried out efficiently.

\section{(c) Link-Layer Issues}

These networks involve high level computing which cannot be managed properly with the existing Media Access Control (MAC) protocols. Therefore, new MAC protocols are needed for wide-ranging channel choice with improved security [17].

\section{(d) Routing}

For UAV networks, an effective and more strong routing protocols are needed which can deliver higher transmission rates and minimum delays during operations, effective route, quick retrieval to the client [16-19].

\section{(e) Strength Managing and Supremacy}

Every UAV contains low backup energy. Thus, well organized designs and outlines are needed to ensure less utilization of involved energy units.

\section{(f) Channel Security}

Flooding, masquerade attacks; black holes, node impersonation, and malicious code are some security threats possible in networks which require effective approaches to manage any such issues or misuse in arrangement.

\section{(g) Uses and Safeguarding}

When the network is ready for a deployment, it will not be complex and perform operation easily. The price and period for the maintenance and deployment must be low [29, 31].

\section{NETWORK DESIGN}

Unmanned aerial vehicle (UAV) works independently or it can function in incorporation with other networks by way of multi ad hoc creation through the satellite communication, which provides operative coordination between the connected network nubs. Different transmission bands are selected according to the requirement and available resources. [20-21].

Fig. 5. shows, the network design comprising different flying nodes connecting each other and with the satellite plus at the same time satellite communicating to the ground station.

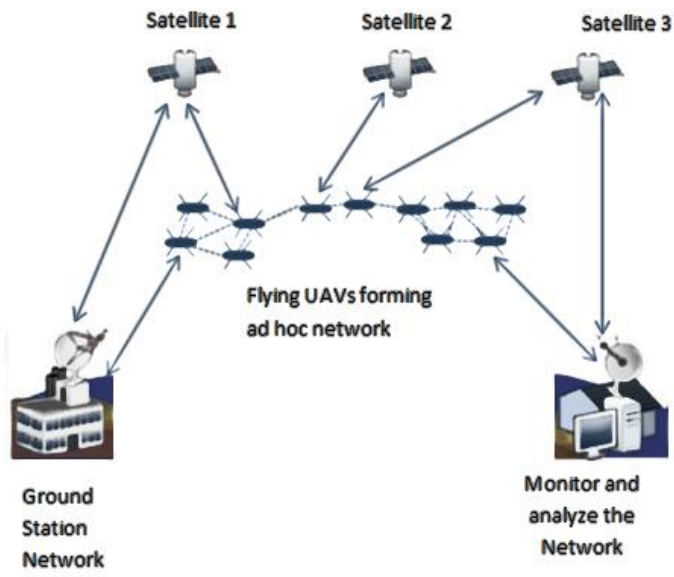

Fig. 5. Complete Network Design

\section{CLASSIFICATION OF UNMANNED AERIAL VEHICLE NETWORKS}

There are various mechanical discrepancies in functioning of UAV networks like (a) latency, (b) scalability, plus (c) adaptability. Latency can be a major factor in failures of the UAV networks [1, 22]. The more nodes the larger is the network coverage, as the space linking the nodes decreases and the chances of nodes traversal increases [34, 23].

Scalability decides the boundary of the networks up to how far network can be covered [10, 11, 27].

According to these characteristics, we can arrange UAV networks in two groups (i) Single UAV arrangement and (ii) multi-UAV arrangement (Fig. 6).

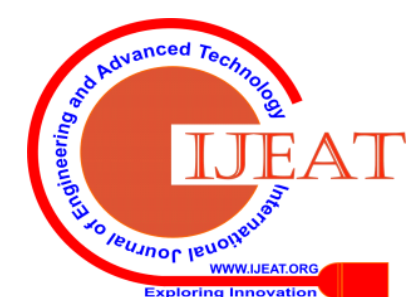




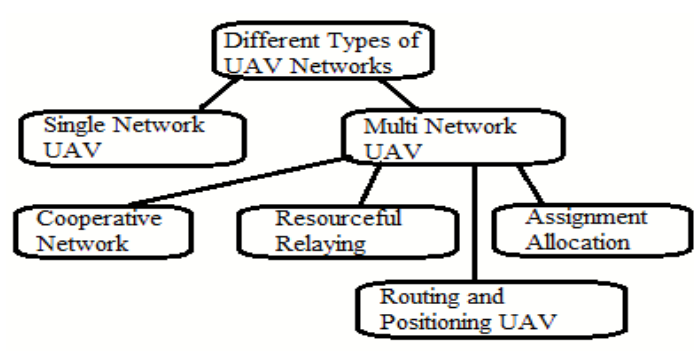

Fig. 6. Taxonomy of unmanned aerial vehicle networks.

\subsection{SINGLE (UAV)}

This system was initially developed for the purpose of navigation and monitoring the remote objects and consisted of flying vehicle requiring human control but these days with the development of drones their area of application have been increased. Single UAV can consist of ' $n$ ' number of UAVs where each node works independently without any types of intra and inter-network connection. Doitsidis et al. [25] have planned a structure that was centered on a fuzzy logic which could do navigation with the help of single UAV.

Rubin et al. [26] suggested structure focused on backbone networks for data transfer in groups of networks. The basis of this network was spatial power control algorithm (SPCA) using networking concept to detect and correct the collision in the network. Palazzi et al. [24] suggested a prototype that used HAPS as satellite for monitoring in metropolitan areas. Using HAPS/UAVs in places of hotspot could allow transmission control protocol (TCP) for sharing of data. Palazzi and Roseti et al. [29] further developed a connection between HAPS and satellite communication which enhanced the transportation.

\subsection{MULTI-(UAV)}

Multi-UAVs are intricate structures that have a capability of managing complicated issues because of their dynamic and efficient framework. It consists of ' $n$ ' number of UAV connection between the nodes but with no interference, and each node knows the place and strategy of the other node.

Chandler et al. [31] explained the concern about the cooperative mechanism in the sovereign flying nodes using stream of auction algorithms.

Flint et al. [30] explained the issues regarding the creation of pathway for synchronized functioning between the multiple nodes and used dynamic programming as a solution.

Vincent and Rubin [28] suggested architecture of swarm intelligence for cooperative network that performed organized aerial functions.

The sub categories of multi-UAV can be discussed as follows:

\subsection{COOPERATIVE NETWORKS}

This term explains the synchronized functioning of the UAVs to do complicated functions. In this mode the UAVs can form a static or a dynamic network. Various network models have been designed based on the cooperative nature of the UAVs. Polycarpou et al. [32] suggested an architecture where cooperative mechanism can be used by dispersed vehicles with controlled functioning.

Bekmezci et al. [33] discussed the complete structure of cooperative networks in example of flying ad hoc network (FANET) and the already implemented technologies such as Mobile ad hoc network (MANETs) / Vehicular ad hoc network (VANETs).

\subsection{RESOURCEFUL RELAYING}

Relaying can allow the creation of temporal networks that is capable of handling sudden break down as in MANET / VANET. However, in multi-UAV system opportunistic relaying is needed that is capable of enhanced utilization of resources.

Hauert et al. [5] suggested a UAV swarm for resourceful relaying using small-aerial nodes known as SMAWNET, which was based on an artificial intelligence approach which in the present world formed the basis of flying ad hoc network (FANET).

Cetin et al. [34] suggested aerial vehicle for cooperative relaying to prevent some collisions among moving obstacles. This method was based upon the formation of potential fields for the identification of hurdles.

\subsection{ASSIGNMENT ALLOCATIONS}

Assignment allocation can be applied to the vehicles having capability of recognizing maneuver decisions for numerous assignments including collision avoidance and cooperative solution for complex task.

Liu et al. [36] suggested UAVs analysis on rational pathway establishment for complex task related to surveillance. This approach is based on computation of possibility map for target gathered data.

Asher et al. [35] suggested the setup for UAVs in an ad hoc network communication that was created for certainty and control. There is some algorithm which is used for handling cooperative task in UAV network, such algorithm is Randomized based algorithm (RBA) tree.

\subsection{ROUTING AND POSITIONING UNMANNED AERIAL VEHICLE}

In heterogeneous networks, directing can always be an interesting task in case of UAV-guided networks. When two or more networks are connected a common interface it more difficult to support data forward to the desired nodes. Most of the researchers are working on that networks and try to find a better solution for UAV networks. Our main focus is to study over vibrant ad hoc network routing by using UAV.

Iordanakis et al. [37] studied various routing plans for flying networks in terms of ad hoc network. The authors are explaining the various plans that are based on infrastructure routing and cannot be implemented for UAV network.

Zang et al. [38] suggested a clustering algorithm with the help of UAV networks. The author is explaining the mobility forecast algorithm. This algorithm is based on the proposed computation established or described how long the connection can be established between the two nodes.

\section{RESULT}

Integrated architectures have increased the areas of applications of networks, such as exploring cyclones, acting as spy bot in difficult operation, distant sensing, health monitoring at remote areas etc. Cooperation among different networks can be established with the use of many frameworks and models. Different cooperative approaches using UAVs have been considered in this paper. The detailed study and comparison of these approaches have been discussed. FANETs have more controlled mobility, 
simplicity of upgrading and deploying, improved connectivity and higher transfer rates as compared to the prevailing networks.

\section{CONCLUSION}

In multi-UAV system, data sharing is important to transmission and control algorithm. The system contains various designing problems in comparison to single UAV. It solves many problems related to scrutiny, direction finding as well as exploration. Cooperation is an important aspect which functions in synchronization with other ad hoc networks to achieve the efficient architectures and frameworks that could be useful for attaining coordination.The efficient and cooperative frameworks solve the problem such as communal functioning of diverse networks, between the ground and the aerial network. In this paper, the prevailing UAV architecture have separately probed or evaluated to form cooperative networks. There are multiple factors and segments that are acknowledged based on their operability in cooperative network creation. Also the design factors for their synchronization in the individual networks have been discussed. In addition, different issues, usages and structural

\section{REFERENCES}

1. Sharma V, Kumar R. A cooperative network framework for multi-UAV guided ground ad hoc networks. Journal of Intelligent \& Robotic Systems 2015; 77(3-4):629-652.

2. Bekmezci l, Sahingoz OK, Temel A. Flying ad-hoc networks (FANET): a survey. Ad Hoc Networks 2013; 11(3):1254-1270.

3. Sharma V, Kumar R. An opportunistic cross layer design for efficient service dissemination over flying ad hoc networks (FANETs). In 2015 2nd International Conference on, Electronics and Communication Systems (ICECS): IEEE, Coimbatore, India, 2015; 1551-1557.

4. Hauert S, Zufferey J-C, Floreano D. Evolved swarming without positioning information: an application in aerial communication relay. Autonomous Robots 2009; 26(1):21-32.

5. Yang Y, Minai A, Polycarpou MM. Evidential map-building approaches for multi-UAV cooperative search. In Proceedings of the American Control Conference, Vol. 1, Portand Hilton and Towers, Portland, 2005; 116.

6. Zhang G, Yang K, Liu P, Feng X. Incentive mechanism for multiuser cooperative relaying in wireless ad hoc networks: a resource-exchange based approach. Wireless Personal Communications 2013; 73(3):1-19.

7. Sharma V, Kumar R. Service-oriented middleware for multi-UAV guided ad hoc networks. IT Convergence Practice (INPRA) 2014; 2(3):24-33.

8. Medina D, Hoffmann F, Ayaz S, Rokitansky C-H. Feasibility of an aeronautical mobile ad hoc network over the North Atlantic corridor. In 2008.SECON'08. 5th Annual IEEE Communications Society Conference on Sensor, Mesh and Ad Hoc Communications and Networks: IEEE, San Francisco, CA, 2008; 109-116.

9. Sakhaee E, Jamalipour A, Kato N. Aeronautical ad hoc networks. In Wireless Communications and Networking Conference, 2006.WCNC 2006. IEEE, Vol. 1: IEEE, Las Vegas, NV, 2006; 246-251.

10. JP, Fraser C, Kulling KC, Bertuccelli LF, Toupet O, Brunet L, Bachrach A, Roy N. Increasing autonomy of UAVs. Robotics \& Automation Magazine, IEEE 2009; 16(2):43-51.

11. Lilien LT, Othmane LB, Angin P, DeCarlo A, Salih RM, Bhargava B. A simulation study of ad hoc networking of UAVs with opportunistic resource utilization networks. Journal of Network and Computer Applications 2014; 38:3-15.

12. Lilien L, Gupta A, Kamal Z-E, Yang Z. Opportunistic resource utilization networks-a new paradigm for specialized ad hoc networks. Computers \& Electrical Engineering 2010; 36(2):328-340.

13. Amazon (Available from http://www.amazon.com/b? node=8037720011.) [last Accessed on 18 December 2019]

14. Santi P. Topology control in wireless ad hoc and sensor networks. ACM Computing Surveys (CSUR) 2005; 37(2):164-194. obligation have also been conferred.

15. Rajaraman R. Topology control and routing in ad hoc networks: a survey. ACM SIGACT News 2002; 33(2):60-73.

16. Tarique M, Tepe KE, Adibi S, Erfani S. Survey of multipath routing protocols for mobile ad hoc networks. Journal of Network and Computer Applications 2009; 32(6):1125-1143.

17. Ramanathan R, Redi J. A brief overview of ad hoc networks: challenges and directions. IEEE communications Magazine 2002; 40(5):20-22.

18. Boukerche A, Turgut B, Aydin N, Ahmad MZ, BlniL, Turgut D. Routing protocols in ad hoc networks: a survey. Computer Networks 2011; 55(13):3032-3080.

19. Conti M, Giordano S. Mobile ad hoc networking: milestones, challenges, and new research directions. Communications Magazine, IEEE 2014; 52(1):85-96.

20. Zhou L, Hu R, Qian Y, Chen H-H. Energy-spectrum efficiency tradeoff for video streaming over mobile ad hoc networks. IEEE Journal on Selected Areas in Communications 2013; 31(5):981-991.

21. Bae C, Stark WE. A tradeoff between energy and bandwidth efficiency in wireless networks. In Military Communications Conference, MILCOM-IEEE, Orlando, FL, USA, 2007; 1-7.

22. Gu DL, Pei G, Ly H, Gerla M, Zhang B, Hong X. UAV aided intelligent routing for ad-hoc wireless network in single-area theater. In Wireless Communications and Networking Conference, 2000.WCNC. 2000, Vol. 3: IEEE, Chicago, IL, 2000; 1220-1225.

23. Li W, Cheng X, Jing T, Xing X. Cooperative multi-hop relaying via network formation games in cognitive radio networks. In IEEE INFOCOM, Turin 2013; 971-979.

24. Palazzi CE, Roseti C, Luglio M, Gerla M, Sanadidi MY, Stepanek J. Satellite coverage in urban areas using unmanned airborne vehicles (UAVs). In Vehicular Technology Conference, 2004. VTC 2004-Spring, Vol. 5:2004 IEEE 59th, Los Angeles, CA, USA, 2004; 2886-2890.

25. Rubin I, Behzad A, Ju H-J, Zhang R, Huang X, Liu Y, Khalaf R. Ad hoc wireless networks with mobile backbones. Personal, Indoor and Mobile Radio Communications, 2004. 15th IEEE International Symposium on PIMRC 2004, Vol. 1, Barcelona, Spain, 2004; 566-573.

26. Doitsidis L, Valavanis KP, Tsourveloudis NC, Kontitsis M. A framework for fuzzy logic based UAV navigation and control. In 2004 IEEE International Conference on, Robotics and Automation, 2004 Proceedings. ICRA'04, Vol. 4, Tampa, FL, USA, 2004; 4041-4046.

27. Yang Y, Polycarpou MM, Minai AA. Multi-UAV cooperative search using an opportunistic learning method. Transactions- American Society of Mechanical Engineers Journal of Dynamic Systems Measurement and Control 2007; 129(5):716

28. Vincent P, Rubin I. A framework and analysis for cooperative search using UAV swarms. In Proceedings of the 2004 ACM symposium on Applied computing: ACM, Nicosia, Cyprus, 2004; 79-86.

29. Palazzi CE, Roseti C, Luglio M, Gerla M, Sanadidi MY, Stepanek J. Enhancing transport layer capability in HAPS satellite integrated architecture. Wireless Personal Communications 2005; 32(3-4):339-356.

30. Flint M, Polycarpou M, Fernandez-Gauche and E. Cooperative control for multiple autonomous UAV's searching for targets. In Proceedings of the 41st IEEE Conference on in Decision and Control, 2002, Vol. 3, OH, USA, 2002; 2823-2828.

31. Chandler PR, Pachter M, Swaroop D, Fowler JM, Howlett JK, Rasmussen S, Schumacher C, Nygard K. Complexity in UAV cooperative control. In American Control Conference, 2002. Proceedings of the 2002, Vol. 3, Anchorage, AK, USA, 2002; 1831-1836

32. Polycarpou MM, Yang Y, Passino KM. A cooperative search framework for distributed agents. In Intelligent Control 2001; 2001(ISIC'01):1-6.

33. Sahingoz OK. Networking models in flying ad-hoc networks (FANETs): concepts and challenges. Journal of Intelligent \& Robotic Systems 2013; 74(1-2):1-15.

34. Cetin O, Zagli I, Yilmaz G. Establishing obstacle and collision free communication relay for UAVs with artificial potential fields. Journal of Intelligent \& Robotic Systems 2013; 69(1-4):361-372.

35. Asher YB, Feldman S, Gurfil P, Feldman M. Distributed decision and control for cooperative UAVs using ad hoc communication. IEEE Transactions on Control Systems Technology 2008; 16(3):511-516.

36. Minjia L, Jie L, Yuyu Y. Research of UAV Cooperative Reconnaissance with Self-Organization Path Planning. Atlantis Press: Beijing, China, 2013. 
37. Iordanakis M, Yannis D, Karras K, Bogdos G, Dilintas G, Amirfeiz M, Colangelo G, Baiotti S. Ad-hoc routing protocol for aeronautical mobile ad-hoc networks. In Fifth International Symposium on Communication Systems, Networks and Digital Signal Processing (CSNDSP), Patras, Greece, 2006;

38. Zang C, Zang S. Mobility prediction clustering algorithm for UAV networking. In GLOBECOM Workshops (GC Wkshps), 2011 IEEE: IEEE, Houston, TX, 2011; 1158-1161.

\section{AUTHORS PROFILE}

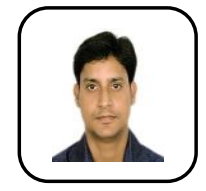

Sandeep Raj, Research Scholar from Al-Falah University, Faridabad Haryana, India. Received M. Tech degree in CSE (2006-2008) from Centre for Development of Advanced Computing (C-DAC NOIDA), affiliated to GGSIPU, Delhi. B. Tech in CSE (2001-2005) from Ambedkar Institute of Technology affiliated to GGSIPU, Delhi. His area of interest is Flying Ad hoc networks (FANETs) and Wireless sensor networks (WSN). He has twelve years of experience in the field of teaching and research. Attended many Faculty Development programs (FDPs) and Workshops.

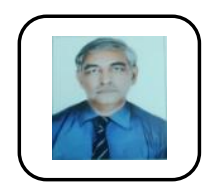

Dr. V.K. Panchal is professor at Al-Falah University, Faridabad, Haryana, India. Former Associate Director at Defense Terrain \& Research Lab, Defense and Research Development Organization (DRDO), Delhi. He is an Associate Member of IEEE (Computer Society) and Life Member of Indian Society of remote sensing. He served the Indian society of remote sensing Delhi chapter as vice-president. He has done Ph.D. in computational intelligence from Jawaharlal Nehru university (JNU), Delhi and M.S in Information Systems from BITS, Pilani. His research interests are in the synthesis of terrain understanding model based on incomplete information set using bio-inspired intelligence and remote sensing. He has chaired sessions \& delivered invited talks at many national \& international conference.

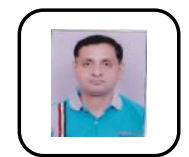

Dr. Rajiv Chopra is an Associate Professor in CSE Deptt. at Guru Tegh Bahadur Institute of Technology (Aff. to GGSIPU, Delhi). He has vast experience of 20 years. He has authored 30 books for the students of graduate and post-graduate students. The author has 30 research publications in International Conferences and in Journals that are refereed. He has attended 28 workshops, FDPs and seminars at both International as well as National levels. He is endowed with the rare traits of strong knowledge of fundamental and the ability to combine intelligence with imagination. Based on the positive feedbacks of his other books by the readers (students and faculties) one can affirm his dexterity and sharp intellect. Apart from his good academic standing, the author has following achievements/awards to his credit: -1 . Life Member (LM) of COMPUTER SOCIETY OF INDIA (CSI).2. Certification on 'Ethical Hacking' from Crezone Technologies Delhi, CSI, India.3.MS AZURE-CLOUD CERTIFIED on 30.04.2016.4. CEGR Certificate Research from 20/09/2017. 5.Honored by IARDO academic Excellence Award 2019 for the Best College Teacher of the year 7th December 2019 at Mumbai.

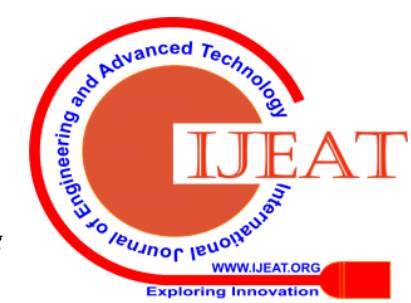

